\title{
Contingencia e identidad: retos para un diálogo transdisciplinar entre la historiografía y la didáctica de la historia
}

\begin{abstract}
Resumen
En el presente trabajo pretendemos analizar las relaciones entre la historiografía y la didáctica de la historia en el caso español, partiendo de una propuesta fenomenológica sobre los múltiples agentes que conforman las conciencias históricas en las sociedades contemporáneas. La atonía principal entre el conocimiento histórico y su transmisión en las aulas se sitúa en torno a dos conceptos: la identidad y la contingencia. En primer lugar, la historia mantiene en el sistema educativo una función nacionalizadora que obvia la historiografía moderna sobre la temática, ahondando en su condición de imaginario social construido e histórico. El currículo de enseñanza continúa caracterizado por un sentido teleológico y positivista de la historia patria como sucesión de hechos que explicarían el viaje en el tiempo de un ente personificado y homogéneo: la nación. En segundo lugar, analizamos la continuidad de las concepciones deterministas y teleológicas de la historia, que a partir de esquemas causales cerrados pretenden explicar acontecimientos complejos y contingentes. Tanto la función identitaria de la enseñanza de la historia como la noción positivista de la disciplina proyectada a un fin ineludible están en el origen de la historiografía moderna y en su integración en los sistemas educativos de los Estados nación. De ahí que sea necesario reflexionar sobre los usos del pasado e integrar la epistemología, la historiografía y nociones sobre la elaboración del conocimiento histórico y la contingencia en todos los niveles del sistema educativo.
\end{abstract}

Palabras clave: Historiografía. Didáctica de la Historia. Identidad. Contingencia. Progreso.

\author{
Cesar Rina Simon \\ Doctor en Historia Contemporánea por la \\ Universidad de Navarra. Profesor de la \\ Universidad de Extremadura. \\ ESPANHA \\ cesrina@unex.es \\ orcid.org/0000-0002-1825-0097
}

\section{Juan Luis de la Montaña Conchiña}

Doctor en Historia Medieval por la Universidad de Extremadura. Profesor de la Universidad de Extremadura. ESPANHA jmontana@unex.es orcid.org/0000-0003-0569-1501

\section{Para citar este artículo:}

RINA, Cesar; DE LA MONTAÑA, Juan Luis. Contingencia e identidad: retos para un diálogo transdisciplinar entre la historiografía y la didáctica de la historia. Tempo e Argumento, Florianópolis, v. 11, n. 26, p. 287 -317, jan./abr. 2019. 


\title{
Contingency and identity:
} challenges for a transdisciplinary dialogue between historiography and didactics of history

\begin{abstract}
In the following theoretical work we intend to analyse the relationships between historiography and the didactics of history in the Spanish case starting from a phenomenological proposal about the multiple agents making up historical consciousness in contemporary societies. The main atony between historical knowledge and its transmission in the classroom is situated around two concepts: identity and contingency. In the first place, history maintains a nationalizing function in the educational system that obviates modern historiography on the subject, delving into its condition as a constructed and historical social imaginary. The teaching curriculum goes on characterized by a teleological and positivist sense of the country's history, as a succession of events that would explain the journey in time of a personified and homogenous entity: the nation. Secondly, we analyse the continuity of the deterministic and teleological conceptions of history, which, based on closed causal schemes, try to explain complex and contingent events. Both the identity function of the teaching of history and the positivist notion of the discipline projected to an inescapable end are at the origin of modern historiography and its integration into the educational systems of nationstates. Hence, it is necessary to reflect on the uses of the past and to integrate epistemology, historiography and notions about the elaboration of historical knowledge and contingency at all levels of the educational system.
\end{abstract}

Keywords: Historiography. Didactics of History. Identity. Contingency. Progress. 


\section{Introducción}

¿Por qué el sistema educativo español -aunque la cuestión es extensible al de cualquier otro estado- obliga al alumnado de enseñanza primaria y secundaria a cursar asignaturas relativas al pasado? ¿Por qué la compresión y el conocimiento de acontecimientos y procesos pretéritos adquieren una relevancia semejante en los currículos al de las habilidades lingüísticas o matemáticas? Estas preguntas son especialmente significativas si tomamos en consideración la deriva utilitarista de los principios ideológicos y pedagógicos que determinan los fines de la educación. Nuestro objetivo en este artículo es abordar en un horizonte teórico estas cuestiones desde la perspectiva epistemológica de la disciplina histórica y entablar un diálogo con su enseñanza.

Rüssen (2006) ha destacado la importancia de la didáctica de la historia como disciplina intermediaria entre la historiografía y la transmisión y perpetuación de imaginarios sociales sobre el pasado. La escuela es un centro, no el único, donde se perpetúan, cuestionan o complementan los contenidos de la memoria colectiva. La didáctica de la historia es un nexo fundamental al que generalmente la historiografía ha prestado escasa atención, lo que en cierta manera permite explicar su afonía y la desconexión entre las conclusiones del trabajo de investigación y su transmisión educativa. Sin embargo, como disciplinas autónomas, tampoco se puede exigir que la didáctica sea mera correa de transmisión de la historia académica a las aulas, pues sus fines y métodos abarcan espacios diferenciados.

\section{Fenomenología de la conciencia histórica}

La conciencia histórica del alumnado no solo se forja en el sistema educativo. Esto es fundamental a la hora de tomar en consideración los límites y potencialidades de la historia enseñada y sus usos públicos en la construcción del pensamiento histórico y los modelos de hegemonía contemporáneos (CASPISTEGUI, 2003; DUMOULIN, 2003). Tenemos acceso al pasado a partir de agentes y mecanismos generadores de conciencia histórica (KLEIN, 2013). La historia como disciplina académica y educativa ha perdido la 
función terapéutica -si aceptamos que alguna vez la tuvo- de convocar el futuro mirando al pasado, al tiempo que los contenidos volcados en el currículo responden a patrones anacrónicos. Como ha señalado Zermeño (2010), la historiografía se convirtió, en la modernidad, en una práctica cultural que creó un nuevo sentido de temporalidad fundado en la escritura y constituyó una memoria histórica y una fórmula que orientó el recuerdo de las sociedades hacia determinadas posiciones ideológicas y nacionales. Sin embargo, la disciplina en las últimas décadas, y en el contexto de los tiempos líquidos conceptualizados por Bauman (RINA, 2012), se ha alejado de las explicaciones globales para centrarse en fragmentos (ZERMEÑO, 2016), que no puede interpretarse como una pérdida voluntaria de la centralidad en la creación de la conciencia histórica, pero sí manifiesta una pronunciada atonía entre la historia investigada y la historia enseñada. En las sociedades contemporáneas hiperconectadas y sobreinformadas, la comprensión del tiempo adquiere múltiples dimensiones, y el pasado se forja en la memoria individual a partir de la interacción constante entre aprendizajes formales y no formales.

En este marco, planteamos una articulación fenomenológica abierta y flexible a la multiplicidad de agentes y espacios que conforman las culturas históricas en el presente. Exponemos una teoría con seis tipos o modelos de creación y difusión de conciencia histórica, tomando las siguientes consideraciones previas: los parámetros están interconectados y en ningún caso representan cajones estancos; cada subgrupo es heterogéneo y sus límites son discutibles; y no se trata de una estratificación del tiempo de tipo braudeliano, con base en duraciones longitudinales, sino de una disposición horizontal, sincrónica, de diferentes concepciones y formas de acercamiento al pasado. Tampoco pretendemos jerarquizar unos modelos sobre otros. Estas seis vías se yuxtaponen, se contradicen y se complementan, lo que no las invalida como modelos sintéticos que contribuyen a la comprensión del espacio que ocupa la historia y la didáctica de la historia en la configuración de los imaginarios del pasado. ${ }^{1}$

\footnotetext{
${ }^{1}$ Agradecemos a Javier Fernández Sebastián y a Guillermo Zermeño las acotaciones terminológicas.
} 
1. Pasado estudiado: Lo conformaría la historia enseñada en las aulas y el canon literario e historiográfico que determina lo que es susceptible de ser estudiado por su nivel de relevancia o por su condición de hito nacional. Este pasado selecciona e integra en el canon nacional -o regional o local- determinados acontecimientos y personajes. Las pugnas políticas por definir el currículo de historia constatan la importancia de este espacio de legitimación histórica de las culturas políticas. Este pasado-presente puede ser independiente de la producción historiográfica académica y se configura como conocimiento formal.

2. Pasado mediático: De amplias dimensiones en las sociedades contemporáneas, es refrendado de manera constante en los medios de comunicación y en las redes sociales. Estos pasados están condicionados por contextos determinados y tienen un papel muy relevante en la gestión de la memoria y del olvido. Así mismo, no viene jerarquizado por la acción profesional de los "notarios del pasado", sino que depende de otros factores como la espectacularidad o los índices de audiencia. Entrarían en este subgrupo periódicos y noticiarios de televisión.

3. Pasado normativo: Es el que mana de la función legislativa del Estado a la hora de articular el currículo escolar o las leyes de la memoria histórica. Se trata de un pasado fijado por ley (RINA; CLEMENTE, 2015).

4. Pasado comercializado: Representado por el turismo, los ciclos conmemorativos, las festividades locales, los mercados medievales o las recreaciones históricas. Se trata de un pasado volcado hacia el ocio, comercializado, capitalizado y consumido.

5. Pasado ficción: Muy potente también en la construcción hegemónica de la cultura histórica. Hablamos de series televisivas (SANTOS UNAMUNO, 2018), películas, documentales, videojuegos o novelas históricas que utilizan el pasado -"basado en hechos reales"- para hacer más atractiva la trama.

6. Pasado académico: Elaborado por la historiografía y condicionado por unos cauces de producción científica y unos métodos específicos de tratamiento del pasado. Este modelo tiene dificultades para visibilizarse. Una de sus claves es que parte de un método y una epistemología, y su pretensión inicial no es la de entretener. 
La popularidad de determinados acercamientos no se ha traducido en un especial interés por el pasado académico o el estudiado en planes de enseñanza ni en proyectos de financiación gubernamental, de ahí que pierdan paulatinamente espacio de influencia en la forja de culturas históricas. Estamos, por tanto, ante una aporía entre el gran interés que despierta el pasado en sociedades sedientas de anclajes -series y películas de ambientación histórica, museos, patrimonialización y turistificación-, al tiempo que el historiador encuentra cada vez más dificultades para entrar en las redes de producción y divulgación del pasado. De tal forma, el auge del pasado ha coincidido con la afonía del historiador y su desconexión con la enseñanza del pasado. Dicha crisis no es novedosa y representa una autopercepción permanente de la historiografía al verse incapaz de monopolizar los mecanismos de producción del pasado. Ya lo apuntó Huizinga (1934) en una conferencia apologética de la ciencia histórica dada en Santander, donde criticó la “historia perfumada” y se preguntó si el interés por una "Historia demasiado adornada no significa una debilitación del juicio y una degeneración del gusto, una indolencia espiritual" de un público criado en la era del cine. Huizinga tiraba de nostalgia para inventar un pasado de lectores cultos frente a un presente dominado por la banalización del pasado y su democratización en públicos lectores más amplios.

La noción de crisis es medular a la disciplina en tanto que la historiografía, autorrepresentada como conocimiento científico jerárquicamente superior, no ha sabido interpretar o aceptar su papel compartido con otros ámbitos en la fijación de la cultura histórica. Además, la aceleración del tiempo experimentado, el desarraigo que conllevan los fenómenos de la globalización y la mercantilización del pasado han convertido la historia en una plataforma identitaria y de consumo alejada de su deontología profesional.

Nuestro trabajo teórico se enmarca en las interacciones entre el pasado estudiado y el pasado académico, en el diálogo y los trasvases necesarios entre la historiografía y la didáctica de la historia. Esta relación es más relevante si tomamos en consideración los datos estadísticos que vinculan a la mayoría de los historiadores con la docencia en todos los niveles de enseñanza. La historia investigada precisa de cauces de transmisión, síntesis y explicación y ahí entra en juego la didáctica. 
Sin embargo, la atonía entre ambas disciplinas se constata en dos casos de estudio paradigmáticos: la función nacionalizadora de la historia y la pervivencia de la teleología y de la idea de progreso en la historia enseñada, asentada en un conocimiento positivista que encuentra en el pasado las claves para refrendar el avance social. De esta forma aparece en las aulas el anacronismo que supedita el pasado al presente en una línea narrativa que dirige todo lo ocurrido a un fin determinado. El historiador se ve relegado al pasado académico, con una influencia residual en la conciencia histórica, lo que sitúa a la disciplina en una búsqueda perpetua de utilidad profesional, una vez perdida su función como constructora de los mitos premodernos y modernos. Nos hemos interesado por el proceso de traducción del conocimiento historiográfico al libro de texto, al currículo y a la práctica educativa, que obliga al historiador a posicionarse para seleccionar y tramar el pasado. Ya lo advirtió Gil de Zárate (1855, p. 117) a mediados del siglo XIX: “La cuestión de la enseñanza es cuestión de poder, el que enseña domina, puesto que enseñar es formar hombres, y hombres amoldados a las miras del que adoctrina."

Una de las preguntas que la epistemología historiográfica tiene que responder es cuánta cantidad de pasado nuestras sociedades pueden soportar. En 1874, Nietzsche planteó en Sobre la utilidad y el Perjuicio de la Historia para la vida la posibilidad de liberar al presente del pasado, y Paul Valéry, en un texto crítico a la musealización, señaló que “nuestra herencia nos aplasta”. En 1938, Croce propuso que la historiografía tenía la capacidad de liberarnos de la historia, del exceso de pasado. Bourdieu también ha señalado que es una herramienta de combate contra la fetichización de la historia y su función en la articulación de mitos e identidades (ROBIN, 2003). De ahí que planteemos en este trabajo la necesaria incorporación de la epistemología y la reflexión historiográfica en la didáctica de la historia para romper amarras con las narrativas teleológicas y nacionalistas.

\section{Enseñanza de la historia e identidad}

La primera atonía significativa que identificamos entre la historiografía y la enseñanza de la historia es el papel del conocimiento del pasado en la construcción de las 
identidades contemporáneas. "La historia ocupó -y ocupa- una función preponderante en la forja de los imaginarios nacionales desde su irrupción en los albores del siglo XIX y sus narrativas se extienden hasta el presente" (NORA, 2011, p. 377). Pese a los vaticinios del fin de la historia o del fin de las naciones en el contexto ideológico de la globalización y de la superación del modelo estadocéntrico, la educación continúa perpetuando modelos nacionales que identifican al alumnado con una serie de referencias del pasado que, ordenadas en una trama, concluyen de manera irremediable en el presente de la nación (MIRALLES; GÓMEZ, 2017; MOLINA; MIRALLES; DEUSDAD; ALFAGEME, 2017; López, 2012). El caso más representativo es la inclusión en manuales y en el currículo escolar de Historia de España de acontecimientos anteriores a la formación del Estado nación: La España Romana, La España Medieval, etc. La fragmentación curricular de la enseñanza de la historia en las comunidades autónomas, bien desde una lógica regionalista, bien desde el nacionalismo centrífugo, ha mantenido su función legitimadora del status quo político e identitario en una narrativa del pasado puesta al servicio de las instituciones o de los modelos territoriales (PÉREZ GARZÓN, 2010).

La función nacionalizadora de la historia enseñada entra en conflicto con la trayectoria de la historiografía en las últimas décadas, marcada por el cuestionamiento de las narrativas nacionales desde el constructivismo, la teoría de las comunidades imaginadas, del nacionalismo banal e incluso desde el etnosimbolismo. Se produce, por tanto, un fuerte desajuste entre la narrativa histórica oficial refrendada por las instituciones y reforzada en leyes educativas, rituales conmemorativos o en procesos de patrimonialización, y las conclusiones de la historiografía reciente al respecto. En este sentido, podemos señalar un desajuste entre dos formas contradictorias de comprensión del pasado, en que la afonía o el repliegue de la historiografía ha provocado un preocupante desajuste entre las disciplinas y su enseñanza (RINA, 2017). Este desajuste se vislumbra en la alteridad entre dos modelos que entran en conflicto en el seno de la enseñanza de las humanidades y de las ciencias sociales relativas a la identidad nacional. Al mismo tiempo que se proyecta de modo transversal la superación de modelos educativos patrióticos en aras de fomentar patrones de ciudadanía cosmopolitas, la historia enseñada continúa perpetuando modelos identitarios y de legitimación del 
Estado nación (PÉREZ GARZÓN, 2008). Los esfuerzos por superar el marco Estado nación de la historia en las aulas se ve frenado por la presencia deíctica de contenidos articulados en torno a su pertenencia o no a la nacionalidad (BILLIG, 1995; PARRA; SEGARRA, 2018; LÓPEZ FACAL; SAIZ, 2016, 2012).

Las narrativas identitarias en el sistema educativo se justifican desde su vinculación a la formación de buenos ciudadanos, patriotas, conocedores de la historia nacional y comprometidos con la misma (GÓMEZ; RODRÍGUEZ; MIRALLES, 2015; SAIZ; LÓPEZ, 2016; CARRETERO, ASENSIO; RODRÍGUEZ-MONEO, 2013). De esta manera, la identidad nacional se canaliza en el sistema educativo como un marco, por un lado, lógico y banal y, por otro, como una apelación de compromiso. La nacionalidad supondría una escala superior en la que la individualidad del alumnado se diluye en una causa colectiva superior.

Una de las herramientas fundamentales en la nacionalización por la historia enseñada ha sido la elevación de la disciplina al árbol de las ciencias, equiparable a las ciencias naturales, por la cual, al igual que se podía comprender mediante leyes el mundo natural, las acciones del hombre en el pasado eran susceptibles de reconocerse desde planteamientos metodológicos científicos, e incluso llegar a predecir sus actos. De esta forma, la historia nacional se vistió de narración objetiva y aséptica (FONTANA, 2000), una historia concebida a nivel escolar como conocimiento de hechos y variables objetivas. Este modelo no es coherente con el devenir de la historiografía de las últimas décadas y esconde una función moralizante y un uso público del pasado a partir de su categorización como ciencia. Una historia presentada como verdadera supone una posición acrítica que redunda en los mitos y en la consideración dogmática de lo enseñado, especialmente en lo referente a la identidad nacional o a la confianza positivista en el progreso (CERTEAU, 1993). Esta tendencia contribuye a la perpetuación en las aulas de la escatología de la historia. Así pues, una de nuestras propuestas de actuación es explicar los acontecimientos como fenómenos específicos, es decir, irrepetibles, no se pueden reproducir en condiciones de laboratorio y, por lo tanto, no susceptibles de comprenderse en un plano causal cerrado. 
Uno de los sesgos de la historia enseñada como sustento de identidad es la cosificación llevado a cabo a partir de la patrimonialización, ligada a procesos contemporáneos de auge del turismo cultural y valorización de las identidades locales, regionales o nacionales. Los restos del pasado son utilizados para testificar la antigüedad o prestigio de la comunidad y atraer turistas (GODINHO, 2017). El conocimiento del pasado adquiere, según esta lógica, una función mercantilista, que aporta al turismo materiales susceptibles de ser consumidos (DOSSE, 2000).

\section{Enseñanza de la historia y contingencia}

La segunda atonía que localizamos entre la historiografía y su didáctica es la perpetuación en la legislación educativa, el currículo, los manuales y las concepciones docentes de una historia ligada a la escatología del progreso (SAIZ; GÓMEZ; LÓPEZ, 2018). La explicación de los acontecimientos como suma de evidencias interrelacionadas por ejes causales conlleva el riesgo de conducir la conciencia histórica hacia la teleología y de generar explicaciones finalistas entre el alumnado, obviando que es el historiador el que hace coherente en su discurso lo contingente.

Este modelo de comprensión se remonta a los orígenes de la consolidación de la historia como materia escolar y como disciplina científica, contextos que coincidieron cronológicamente con su profesionalización. La historiografía rankeana, que concebía la historia como la escritura de las cosas tal y como sucedieron, continúa impregnando las diferentes aristas de los contenidos históricos del sistema educativo. Historiadores como Dilthey o Taine contribuyeron a situar a la historia en el plano de las ciencias en un contexto competitivo entre disciplinas por alcanzar dicho estatus. Taine, en el Tratado de filosofía del arte de 1882 (apud ZERMEÑO, 2010, p. 25), apuntó que

el método moderno que yo sigo y que comienza ahora a penetrar en todas las ciencias naturales, consiste en considerar las obras humanas [...] como hechos y productos cuyas propiedades hay que mostrar y cuyas causas hay que investigar. [...] Las ciencias morales tienen que proceder del mismo modo que la botánica [...]. No son otra cosa que una especie de botánica aplicada, sólo que, en lugar de tratar con plantas, tiene que tratar con las obras de los hombres. Este es el movimiento con el cual se van aproximando en la actualidad las ciencias morales y las 
La obsesión disciplinar por la objetivación, por separar al sujeto de la narración del pasado, estuvo en el origen de la inclusión de la historia en el sistema educativo de los Estados nación en formación. La historiografía moderna pensó que era posible reconstruir el pasado desde el origen y sin prejuicios (ZERMEÑO, 2010). "Esta aspiración positivista pretendía convertirla en un conocimiento total cuyos hechos se presentaban entrelazados por relaciones causa-consecuencia que el historiador debía desvelar apoyado en el método científico" (KOSELLECK, 2010, p. 74). Se trata del modelo de Herder, Hegel o Humboldt que se introdujo en el sistema educativo de la mano de la enseñanza de la historia nacional y del que Walter Benjamin (2009), en el seno de la Escuela de Frankfurt, advirtiera en su novena tesis sobre las consecuencias nefastas de la razón instrumental y de la capacidad de la "civilización" para generar "barbarie" en nombre del progreso y la ciencia.

En este sentido, Hannah Arendt (1995) señaló que la historia se dedicaba a la comprensión, diferenciada del conocimiento científico, que generaba resultados unívocos. El pasado, una vez liberado del magistra vitae, no necesita sintetizar ni establecer acuerdos y se erige como un espacio para la confrontación y la crítica, en ningún caso como un conocimiento fehaciente disponible para memorizar, tal y como interpretan las concepciones del alumnado (DE LA MONTAÑA, 2014; GÓMEZ; MIRALLES, 2015). La principal justificación de la presencia de la historia en el sistema educativo sería su capacidad para la comprensión y no su objetividad. De ahí la importancia de trabajar en las aulas cuestiones epistemológicas más que series cronológicas (DE LA MONTAÑA, 2015, 2016). La historia no puede enseñarse como relato rankeano frío y acrítico, sino como conocimiento elaborado por historiadores en diálogo con otros niveles de comprensión del pasado y en contextos determinados.

La contingencia, el espacio de lo posible, tiene un amplio recorrido en la historia de la filosofía. Sin embargo, desde el aristotelismo ha sido apartada del conocimiento científico porque no ofrece explicaciones racionales ni somete los acontecimientos a lógicas causales. El cristianismo ahondó en el rechazo de la contingencia al concebir la 
historia de la humanidad como el resultado de un plan divino predestinado, en que nada era azaroso ni escapaba de la voluntad de Dios. La historia de la humanidad adquirió un sesgo determinista volcado a un fin. El racionalismo moderno sustituyó la teología por la ciencia natural, pero mantuvo el finalismo histórico (NISBERT, 1996). Spinoza o Leibniz retomaron la problemática de la contingencia, relacionándola con el libre albedrío (ROLDÁN; MORO, 2009). Sin embargo, el siglo de la historia estuvo protagonizado por el historicismo y el positivismo, dos formas de comprender el tiempo legitimadas por la razón. Vico, Ranke, Hegel, Kant, Comte, Marx, Herder o Voltaire pensaron que la historia sucedía por algo determinado y que tenía su propia dirección. Montesquieu localizó en De l'esprit des lois (1748) estos determinantes en las características materiales y climáticas que condicionaban la historia y los rasgos de las civilizaciones, como también lo haría Henry Thomas Buckle en History of Civilization in England (1856) buscando leyes fijas y parámetros científicos: "toda generación demuestra que hay algunos hechos regulares y previsibles que la generación anterior había calificado de irregulares e imprevisibles." Adam Smith y la escuela escocesa encontraron la dirección de la historia en la mano invisible que regulaba la economía. También Kant desarrolló el determinismo en Ideas para una historia universal en clave cosmopolita (1784) en que defendió que

las manifestaciones de la voluntad en los actos humanos están determinadas, como todos los restantes acontecimientos externos, por leyes universales de la naturaleza [... ]. Cuando se examina el juego de la libertad de la voluntad humana a la gran escala de la historia universal se descubre un paso regular en sus movimientos [... ] Poco imaginan los hombres en particular, y hasta las naciones enteras, mientras persiguen sus propios fines $[. .$.$] que marchaban inconscientemente bajo la guía de un fin de la naturaleza que$ le es desconocido.

La tarea de la historia para Kant era "intentar descubrir un propósito en la naturaleza tras este disparatado curso de los acontecimientos, y decidir si es al fin posible formular en términos de un plan definido de la naturaleza y la historia de unas criaturas que actúan sin un plan propio." Hegel llevó más lejos el planteamiento kantiano y, en Lecciones de filosofía de la historia (1830), defendió una teodicea para la historia, con una dirección que conduce a una escatología y siempre es progresiva, y se propuso eliminar la 
contingencia de la reflexión sobre el pasado. La historia tenía un designio, un fin último que la disciplina debía de reconocer. "Tenemos que introducir en la historia la creencia y la convicción de que el reino de la voluntad no está a merced de la contingencia." La voluntad superior era la razón: "el presupuesto básico que ella gobierna el mundo y la historia es un proceso racionalmente comprensible" (HEGEL, 1992). La historia se mueve por unas fuerzas superiores que los hombres desconocen, utiliza "al individuo como mero instrumento en beneficio de su propio progreso." Y concluye: "la filosofía debe ayudarnos a entender que el mundo real es como debe ser. [...] El mérito de los individuos se mide por el grado en que reflejan y representan el espíritu nacional." De Hegel partirían múltiples líneas teleológicas de pensamiento, desde el positivismo de Comte en Curso de filosofía positiva (1830-1842), en que dividía la historia en tres estadios progresivos, hasta el marxismo con sus etapas evolucionistas encaminadas hacia el comunismo.

Para todos ellos la historia tenía un sentido, una dirección, un final, como lo tenía para el cristianismo. La historia vendría a suplir al profeta y a la escatología cristiana en un nuevo entramado teleológico y positivista. Al mismo tiempo, las ciencias naturales se consolidaron a partir del rechazo de lo accidental, que sería una forma de reconocer ciertas limitaciones. La utopía de la ciencia positiva pretendía reconocer las leyes naturales que regulaban la totalidad del universo. Sin embargo, esta utopía se quebró tras el trauma de la II Guerra Mundial, abriéndose un espacio a la contingencia en todas las ciencias e hizo más complejas las explicaciones causales. La contingencia negaba la teleología, por ello, destaquemos su interés didáctico con el objeto de evitar la perpetuación del determinismo en las aulas y la comprensión de los acontecimientos como resultado de suma de causalidades. No podemos trabajar las causas históricas en el aula como si se tratasen de ingredientes de un plato de cocina, sino como fórmulas de comprensión y acercamiento a un pasado que es abordado necesariamente desde el presente.

A lo largo de la modernidad hasta la segunda mitad del siglo XX, encontramos escasas referencias que cuestionen el determinismo de las explicaciones causales de la historia, y las que hubo se fraguaron en horizontes intelectuales críticos, o bien desde 
posturas reaccionarias, o bien desde alternativas al liberalismo. Uno de ellos fue Thomas Carlyle, autor de On History (1830), que defendía que los hechos no estaban relacionados de manera simple como padres e hijos (FERGUSON, 1998, p. 48). Trevelyan en Clio, a muse and other essays, literary and pedestrian (1913) también fue muy crítico con la pretensión de la historiografía de hacerse pasar por ciencia a costa de renunciar a la narratividad y someterse a reglas arbitrarias de causa-consecuencia. Por su parte, el historicismo desarrollado por Droysen y Dilthey consideró que la labor del historiador era repensar su método y no buscar leyes generales de funcionamiento universal.

En La condición humana, Arendt (2005) abogó -retomando la genealogía de Nietzsche, Freud y Wittgenstein, quienes consideraban que la creencia en la causalidad era una superstición- por acercarse al conocimiento de nuestras sociedades como el resultado de contingencias históricas y en ningún caso como expresión natural, ahistórica o suprahistórica de una voluntad determinista. Rorty (1991) también planteó esta cuestión. Bourdieu (1998) y Foucault ahondaron en la crítica a la historiografía que ideaba acontecimientos absolutos con la idea de mostrarle "a la gente que son mucho más libres de lo que ellos piensan, que ciertos temas que tienen por verdaderos y por evidentes, han sido fabricados en un momento particular de la historia y que esa supuesta evidencia puede ser criticada y construida" (FOUCAULT, 2001, p. 1597). Y continuaba: "mostrar a la gente que un buen número de cosas que forman parte de su paisaje familiar y consideran universal son el producto de ciertos cambios históricos bien precisos." Bourdieu y Foucault confluyeron en la crítica a las construcciones sociales del pasado, entendiendo la historia como un saber del presente (MORO, 2009). En ambos se vislumbra la reacción al estructuralismo y el rescate de la historia como conocimiento falible $y$, por tanto, cuestionable y circunstancial. La inclusión de la contingencia en el currículo escolar supondría una relativización de los esquemas teleológicos que explican la trayectoria de la humanidad y una apertura de análisis al sinfín de consideraciones que escapan de las explicaciones simplistas causa-efecto (BOURDIEU; WACQUANT, 1998).

La historización de conceptos fue proyectada por Koselleck (1979) al incluir en los procesos históricos las tensiones entre el campo de experiencia y el horizonte de expectativas, en el que abre un espacio a la contingencia entre las acciones, las 
expectativas y los resultados. Esto ya había sido apuntado por Raymond Aron (1938) cuando consideró que el azar era más determinante en las acciones de los hombres que la razón. Por ello, Koselleck cuestionó la posibilidad de reducir a explicaciones racionalescausales acciones y expectativas humanas por la radical imprevisibilidad de los acontecimientos. La causalidad simple domestica el pasado en relaciones causa-efecto. Esto no quiere decir que la contingencia se enfrente en relación dialéctica a la necesidad. Simplemente, cabe apuntar que el interés por la contingencia es señalar la capacidad de la historia de haber sido diferente, cuestionando los engranajes explicativos causales más cerrados. Sin embargo, el parámetro quedó arrinconado en el horizonte de las ciencias por su capacidad de perpetuar las escalas explicativas causales (ROHBECK, 2009).

La contingencia no es un tema de debate central en la historiografía, si bien es ampliamente aceptada por la academia a partir de trabajos como los de Paul Ricoeur, especialmente Contingence et rationalité dans le recit (1986). Es una herramienta que permite cuestionar las escatologías de la historia. Nosotros actuamos movidos por una cierta visión de lo posible, de lo que todavía no es pero puede ser, y es ahí donde se bifurcan los caminos en horizontes de expectativas. Podríamos poner innumerables ejemplos, siendo el más significativo para la historiografía la caída de la URSS, acontecimiento que los modelos causales no vaticinaron. Sólo cuando ocurren, la historiografía establece modelos causa-efecto, en palabras de Carlyley “el caos es lo que el historiador va a describir y a ponderar científicamente" (apud FERGUSON, 1998, p. 13).

Para la historiografía moderna fue fundamental la alteridad entre pasado y presente, que hizo del primero "un país extraño", un lugar susceptible de ser estudiado objetivamente con la distancia del presente (LOWENTHAL, 1998). La historia positivista contó la consumación de unos hechos concatenados y lógicos, movidos por el progreso incesante, y susceptibles de ser reconocidos con el método científico (HÖLSCHER, 2014). Las ciencias naturales y sociales propusieron modelos evolutivos y organicistas aplicados a categorías como la nación, el estado, la raza o la civilización. En este contexto, la historiografía moderna surgió ligada a métodos de emulación biologicista, libres de carga subjetiva (HABERMAS, 2007). Estos modelos disciplinares se trasladaron al sistema 
La literatura científica respecto a la opinión del alumnado sobre qué es la historia es abundante. La conclusión más reiterada es que ésta pervive en el imaginario del alumnado con sus significantes más rankeanos: conocimientos de hechos del pasado tal y como ocurrieron. Es por lo que desde la didáctica de la historia se está haciendo hincapié en la necesidad de integrar en el currículo la epistemología y la historiografía adaptadas a cada nivel educativo (PARKES, 2009; DE LA MONTAÑA, 2015). Se trata de convertir la disciplina con sus prácticas, métodos, teorías y conceptos en objeto de estudio con el fin de superar las nociones descriptivas y numerativas del pasado. Por esto, proponemos la inclusión de la contingencia en la didáctica de la historia con el objeto de tornar más complejo el conocimiento del pasado, cuestionar las explicaciones más deterministascausalistas y problematizar el finalismo histórico que perpetúan tanto los manuales como el currículo y la perspectiva del profesorado. El conocimiento del pasado que se aborda en la etapa escolar tiene una trama con un principio y un final bien definidos, con una lógica que empuja a los acontecimientos hacia una dirección irreversible y previsible, que eleva a la categoría de conocimiento científico entramados de causas y consecuencias establecidos por el historiador una vez concluidos los hechos. Es así como una red de efectos conduce la historia al presente lo que no contribuye a desarrollar un pensamiento crítico, no solo sobre el tiempo, sino sobre la elaboración de su conocimiento y los fines de los diferentes planteamientos historiográficos. Esta historia genera en el alumnado la sensación de encontrarse ante una obra completada, incuestionable desde el momento que se concibe como suma causal de factores lógicos.

Sartre explicaba los mecanismos causales en El ser y la nada (1943) tomando como ejemplo el hecho incuestionable de la Toma de la Bastilla en 1789. La importancia del hecho está en su transformación mediante la historiografía en acción decisiva, en su conversión en acontecimiento. "Se historializa al tratar de descifrar la historia a la luz de sus proyectos y de los de su sociedad. En este sentido se puede decir que el sentido del pasado social está perpetuamente en el presente". El relato histórico trataría de explicar la Toma de la Bastilla a partir de modelos de causalidad que en último término la 
convierten en inevitable. En términos similares, Bertrand Russell identificaba el intento de la historia por "descubrir leyes causales que relacionan diferentes hechos, de la misma manera que las ciencias físicas han conseguido descubrir las interconexiones entre los hechos." Este esfuerzo no era el más relevante para Russell: "la mayor parte del valor de la historia se pierde si no nos interesamos por lo que sucede en ella por sí mismo." La causalidad era una reliquia que sobrevivía "solamente porque se supone equivocadamente que es inocuo pero no lo es" (apud CRUZ, 2008, p. 199). También Karl Popper (1967) fue muy crítico con la asimilación de la historia con las ciencias naturales y la colonización del empirismo. El historiador seleccionó los hechos que consideró importantes y los integró en una red de causas y consecuencias, por lo que no se puede objetivar los métodos para excluir al sujeto, al historiador, de su responsabilidad sobre los mismos (POPPER, 1961, 1967). Igualmente, cuestionaba las escalas jerárquicas del conocimiento establecidas en la modernidad, que situaban en la cúspide a aquellas disciplinas consideradas más científicas y las condenaba a combatir por su grado de cientificidad.

Por su parte, Cioran (1988) criticó la idea de progreso e identificó en los usos públicos del pasado mecanismos de legitimación en nombre de patrones causales y teleológicos. También pensó en la vigencia de la disciplina histórica como magister vitae. Conocer las narrativas del pasado no libera a la humanidad de perpetuar sus prácticas y sus errores. Por eso, la historia es una práctica de comprensión social que toma como punto de partida la sospecha. Koselleck (1993) o Manuel Cruz (2012) también han dado por extinta la noción romántica de la historia como conocimiento tutelar y normativo del presente. Para Cruz (Ibid.), de nada sirve el conocimiento del pasado cuando lo utilizamos para refrendar pensamientos previos. La historia debe ser un campo de indagación, de conflicto interpretativo, y no una explicación cerrada del pasado. El cuestionamiento de la historia como magister vitae tiene que ver con el ocaso de las teleologías políticas, la crisis de la idea de progreso y el repliegue de la disciplina. Como ha señalado Hartog (2003) con el concepto de régimen de historicidad, la historia fue fundamental en la modernidad porque estaba vinculada a proyectos de futuro. El nuevo paradigma, según Hartog, sería el presentismo, en el que las líneas de proyección hacia el pasado y hacia el futuro pierden 
su centralidad. La linealidad de la historia y su carácter unívoco o inevitable se ha visto superada por espacios de contingencia y fragmentación. Una vez que el futuro comienza a ser un lugar extraño o del que desconfiar, el historiador pierde su legitimidad al verse incapaz de controlar el tiempo. La función profética que reservaban al oficio de la historia los manuales clásicos de la Escuela de Annales como Apología de la historia, de Marc Bloch, o Combates por la Historia, de Febvre -más abiertos a la contingencia y a lo impredecible que los modelos de larga duración de Braudel- han perdido su espacio central como generadores de cultura histórica en un horizonte de afonía de la historiografía, desplazada de la toma de decisiones sobre los imaginarios del pasado.

Una de las principales limitaciones de las explicaciones causales simples es que pretenden reducir la acción humana al plano de lo racional (ELSTER, 2010, p. 493; MARQUARD, 2000). En el aula, el riesgo es la reducción de la historia a procesos deterministas, en la que cada acontecimiento tiene su función dentro de una narrativa en la que todos los factores pueden integrarse en redes de explicación lógicas. Este planteamiento infravalora factores como la escritura de la historia, el conflicto, la complejidad o la contingencia. La historiografía contemporánea no concibe la existencia de modelos causa-efecto cerrados como en la biología (MACINTYRE, 1980). "Saber que los hombres del pasado tenían expectativas -previsiones, deseos, miedos y proyectostrae consigo la quiebra del determinismo histórico, al reintroducir retrospectivamente la contingencia en la historia" (RICOEUR, 1999, p. 50). En el primer volumen de Tiempo y narración (2013), Ricoeur desengranó la articulación de las narrativas del pasado a partir de la asimilación de acontecimientos en tramas explicativas. La síntesis de estos relatos en modelos causales cerrados obvia la inabarcabilidad del conocimiento histórico y también lo azaroso y lo contingente. Como nos recordaba Arendt (1995, p. 42), solo cuando ha ocurrido algo nos aventuramos a trazar su historia retrospectivamente. Lo ocurrido activa la búsqueda de su pasado, de ahí la incapacidad de la historiografía para establecer leyes generales. Arendt (2004, p. 43) cuestionó también la búsqueda de lo pretérito como ratificación del presente, parque de atracciones o vademécum donde apoyarse para avalar doctrinas o refrendar pautas morales. La historia y su didáctica no 
pueden sustentarse en probar lo evidente o en sintetizar causas y consecuencias, sino en problematizar el sentido del relato historiográfico.

Los contenidos históricos en el sistema educativo se mantienen cercanos a la noción apriorística de finalidad y representan el tiempo como un itinerario en que el presente se valoriza enfrentado al pasado y se analiza en función de su utilidad: legitimidad política, objeto de consumo turístico o sustento identitario (CATROGA, 2009, p. 262). En nuestro abordaje teórico defendemos la inclusión en las aulas de los mecanismos y los debates en torno a la elaboración del pasado que faciliten la comprensión del tiempo no como algo dado y concluso. Como señalara Koselleck (1993), son las expectativas las que median las interpretaciones del pasado. La utopía de la historia moderna se asentó sobre la creencia de que el hombre podía controlar su destino y de que el futuro era el resultado unívoco del pasado. La historiografía intentó sintetizar la complejidad del tiempo bajo criterios científicos de causa-efecto. Sin embargo, es el historiador el que al seleccionar y ordenar los acontecimientos los inscribe en unos ejes con un sentido determinado, de ahí la importancia que tuvo la historiografía y su profesionalización en la forja de las culturas históricas de la contemporaneidad. Por ello, la visión del pasado es dinámica, en diálogo con el presente que la interpreta.

La escuela es el escenario en el que se despliegan las materias, contenidos e ideas acordados socialmente, donde se discierne entre lo que la sociedad debe recordar y lo que debe olvidar. Recordar supone establecer relaciones y buscar la alteridad con un tiempo que ya no es (RICOEUR, 1989). Una de las concepciones más extendidas del alumnado sobre la historia es la comparativa dicotómica entre un pasado de atraso y un presente de progreso. Esta selección y ordenación de pasados no solo corresponde al historiador como hemos visto en el esquema fenomenológico, pero tampoco es responsabilidad individual del alumno. Los imaginarios sociales convocan determinados temas históricos (TODOROV, 2008). Así mismo, la división del tiempo en edades refuerza en el alumnado la idea de una historia volcada al presente-futuro mediante una sucesión evolutiva de etapas y de campos semánticos cualitativos: luces, renacimiento, decadencia, barbarie, progreso, civilización, etc. 
Numerosas investigaciones didácticas de las últimas décadas han incidido en la potencialidad de los esquemas causales en la mejora del aprendizaje de la historia. Esta metodología se justifica como superación del paradigma memorístico y la acumulación de nombres propios y fechas. Sin embargo, por el camino de la causalidad se perpetúa una conciencia histórica positivista y determinista, superada por completo en los horizontes historiográficos. Los argumentos causales cerrados parten de una noción del pasado como un conocimiento completo, de tal manera que se puede deconstruir en sistemas de redes causales que lo explicarían en su totalidad. Este método de enseñar historia deja fuera del alcance del alumno cuestiones epistemológicas fundamentales, como la complejidad de los acontecimientos o el papel del historiador a la hora de establecer rangos de relevancia sobre lo que tiene que ser estudiado e integrado en la narrativa. Una de las vías más recientes en el marco de la didáctica de la historia ha venido de la mano de la aplicación de narrativas como herramienta didáctica, métodos que tampoco han conseguido integrar la elaboración del conocimiento histórico o la contingencia, ni debatir la conciencia histórica teleológica (ORTUÑO; PONCE; SERRANO, 2016; CHAPMAN, 2011, 2013).

\section{Propuesta transdisciplinar: historiografía y didáctica de la historia}

De cara a plantear alternativas a los modelos positivistas y a la función nacionalizadora de la historia en la escuela, así como establecer cauces de diálogo y transferencias entre el pasado enseñado y el pasado académico, consideramos necesario ahondar en una didáctica de la historia que aborde los métodos y la epistemología de la historiografía. Esto sería una herramienta relevante en la superación de la noción de la historia como conocimiento neutro y en su conexión con el presente que la problematiza. Rüssen (2012) define el concepto de pensamiento histórico como el proceso intelectual de creación de sentido sobre la experiencia temporal recurriendo explícitamente a la experiencia del pasado. La historia enseñada debe adquirir una dimensión relacionada con la conciencia del tiempo, su construcción historiográfica, su contingencia y su mutabilidad, pero en ningún caso puede aislar variables o desproblematizar los acontecimientos. Nuestra propuesta pasa por complejizar el conocimiento sobre el 
pasado y no tratar de encorsetarlo en modelos de relaciones causales simples, haciendo hincapié en la contingencia o en el presentismo de la historia. En esta línea, desde la enseñanza de la historia se han aportado interesantes reflexiones sobre la dirección del pensamiento histórico (GÓMEZ, ORTUÑO; MOLINA, 2014; LÉVESQUE, 2008; SEIXAS; MORTON, 2013; VANSLEDRIGHT, 2011).

Las didácticas más comprometidas con el desarrollo del pensamiento crítico inspiradas en la Escuela de Frankfurt- coinciden en la crítica a la pervivencia de la idea de progreso como leitmotiv de la enseñanza de la historia. "Tan pronto como el progreso se convierte en el rasgo característico de todo el curso de la historia, su concepto aparece en un contexto de hipostatización acrítica en lugar de uno de planteamiento crítico" (FEDICARIA, 2014, p. 8). En la práctica docente poco se ahonda en las aporías del progreso y en sus significaciones ideológicas como sistema de legitimidad del horizonte de la modernidad. Walter Benjamin, en El libro de los pasajes, o Adorno y Horkheimer, en Dialéctica de la llustración (2007), explicaron cómo la corrupción teórica de la idea de progreso consistió en pasar de una concepción puesta al servicio de la transformación social a otra de glorificación del presente y su status quo, en la forma de mecanismo discursivo de legitimidad del Estado nación o como vindicación civilizadora del colonialismo occidental.

Nuestra propuesta metodológica pasa por incluir en el currículum referencias epistemológicas e historiográficas que relativicen los escleróticos modelos causales y abran espacio a la contingencia. Una de las posturas más extremas en este sentido, pero con amplias potencialidades en la didáctica de la historia, es el análisis de contrafactuales. En la bibliografía española encontramos algunos trabajos al respecto, si bien es un camino disciplinar muy poco transitado (EVANS, 2017; FERGUSON, 1998). Esta metodología tiene su interés en el aula a la hora de abrir nuevos cauces de comprensión del pasado que contribuyan a cuestionar las narrativas más deterministas, tal y como ha defendido Buxton (2016). La aportación de la contingencia pretende poner el foco en la elaboración del conocimiento del pasado, en la selección, jerarquización y establecimiento de relaciones causales entre los acontecimientos desde el presente. Collingwood, como Croce, consideraba que el historiador sólo podía aspirar a conocer el presente. 
Igualmente, Walter Benjamin (2009) ya advirtió de la frivolidad del pasado como patrimonio o tradición acrítica al servicio del poder. En su tesis XIV afirmó que "la historia es objeto de una construcción cuyo lugar no es el tiempo homogéneo y vacío, sino el que está lleno de tiempo del ahora."

Proponemos una enseñanza de la historia que trascienda de la sucesión de hechos para acercarse al carácter poliédrico y variable de sus relatos, cómo se selecciona el contenido del recuerdo y del olvido y se articulan las narrativas identitarias. También conviene repensar el currículo determinista y la transmisión de los valores asociados a la teleología del progreso para superar la idea del pasado como un lugar arcaizante, mágico, idealizado en términos de atraso o de espacio prístino. Al mismo tiempo, planteamos superponer a la pregunta ¿qué ocurrió?, la manera en que se ha contado, en qué estrategias narrativas ha sido integrado, poniendo el foco en los debates historiográficos más que en la concatenación de referencias, lo que dotaría al alumnado de herramientas críticas (WINEBURG, 2001; GIMENO LORENTE, 2009). De ahí, la importancia de ahondar en la epistemología en el aula para la comprensión de las nociones básicas de la disciplina histórica no como una simple suma de hechos del pasado, sino que estudie el origen, la estructura y los límites del conocimiento histórico en relación con el presente y, por lo tanto, como conocimiento en constante transformación. Esta vindicación es más necesaria en cuanto que ha triunfado en el presente una visión del hombre como cosa entre las cosas -en buena medida por el prestigio de la economía y los modelos cuantitativos y funcionalistas- (SAFRANSKI, 2017, p. 90). El resultado ha sido la confusión entre el tiempo y sus instrumentos de medición.

Por otra parte, sugerimos la incorporación en el currículo de historia de todos los niveles educativos de las teorías modernas del nacionalismo que ahondan en la configuración de sus ideas y superar las lecturas teleológicas, evita la sacralización de un término essencialista y enmarcándolo en determinados contextos históricos. Una de las claves de la consolidación de los Estados nación fue el triunfo cognitivo consistente en la apropiación con exclusividad de la idea de verdad histórica y definirla, regularla y transmitirla mediante el sistema educativo. Urge configurar una nueva didáctica que 
Otra vía aún por explorar en la didáctica de la historia es el estudio del pasado no desde el plano cronológico evolutivo, sino a partir de las transformaciones semánticas y significaciones de los conceptos. Como ha desarrollado Koselleck (2012), no consistiría en analizar la historia desde el discurso, sino de integrar la práctica social con el análisis de los conceptos como agentes históricos. Los conceptos integran las poliédricas e infinitas experiencias del pasado en un marco de lenguaje comprensible. La traducción del pasado al presente se apoya en una serie de estrategias conceptuales que no se han visto reflejadas en el currículo de historia. Por ello, hemos defendido una enseñanza de la historia desde ejes temáticos diacrónicos y conceptos (DE LA MONTAÑA; RINA, 2018).

No cabe duda de que para su implementación sería prioritario transformar los métodos de enseñanza del profesorado en formación de cara a generar nuevas pautas disciplinares. El análisis de los conceptos nos acerca a los principios hermenéuticos de la disciplina: nos invita a reflexionar sobre su estatus, sus metodologías, sus resultados. Priorizamos la historiografía frente a las seriaciones para evitar ahondar en la concepción de la historia como trayecto finalista conducido por leyes causales o por entes colectivos nacionales. Enzo Traverso (2007) ha puesto las cuatro claves que debe surcar la historiografía: contextualización; historicismo crítico; comparativismo; y conceptualización. Estos puntos pueden extenderse a la didáctica de la historia con la superación de la idea de progreso y la historización de los procesos de nacionalización, afrontando la nación como concepto de análisis histórico más que como esencia perennialista; con la inclusión de la idea de contingencia que flexibilice las explicaciones causales; con el acercamiento epistemológico y metodológico a la construcción del relato historiográfico mediante el cual el acontecimiento proyecta una serie de tramas causales que lo explican y determinan; $y$, por último, con el rechazo a un currículo que mantiene a la historia como conocimiento objetivo y apolítico. 


\section{Conclusiones}

Desde un esquema fenomenológico sobre las diferentes vías de formación de las culturas históricas -pasado estudiado, pasado mediático, pasado normativo, pasado comercializado-patrimonializado y pasado académico-, en este artículo hemos abordado la relación entre la historiografía y la didáctica de la historia a partir de dos conceptos en los que se constata una atonía entre la producción académica y el currículo escolar: la historia se presenta como sustento y refuerzo de las identidades nacionales y se muestra acompañada de explicaciones causales deterministas que perpetúan la idea de progreso. Ese modelo de historia, entendido como algo cerrado, lineal y acrítico, es el que aún predomina en la práctica en los entornos escolares.

Una vez descifrado el origen, trascendencia, trayectorias y posibles impactos en contextos educativos de esas dos categorías, hemos planteado una serie de propuestas relativas a la inclusión en todos los niveles educativos de análisis epistemológicos, conceptuales e historiográficos, nociones hermenéuticas más cercanas a la práctica actual de las ciencias sociales y las humanidades, que permitan plantear el trabajo de la historia escolar desde presupuestos radicalmente diferentes. La utopía de la historia de la modernidad, que pretendía reconocer las leyes generales del comportamiento humano, chocó con su radical subjetividad y contingencia. El pasado no es susceptible de ser matematizado ni reducido a lógicas -en no pocas ocasiones simplistas- de causa-efecto, pues no se pueden relacionar los factores como partes de un todo orgánico y finito.

Defendemos la introducción de la contingencia, la epistemología y la complejidad para flexibilizar las escleróticas explicaciones del pasado enraizadas en lógicas causales cerradas. Así mismo, al introducir en la didáctica de la historia el conocimiento de su construcción como disciplina y de parámetros críticos con los usos públicos del pasado, herramientas clave para el trabajo y la construcción del pensamiento histórico, pretendemos resituar el conocimiento de la historia como potente materia formativa, alejándola al mismo tiempo de la función nacionalizadora y de la teleología que mantiene en los sistemas educativos actuales, contribuyendo a la idea de complejizar el acercamiento al pasado y situarlo en el plano de un conocimiento en constante construcción y debate en las sociedades contemporáneas. 


\section{Referências}

ADORNO, Theodor W.; HORKHEIMER, Max. Dialéctica de la Ilustración. Madrid: Akal, 2007.

ARENDT, Hannah. De la historia a la acción. Barcelona: Paidós, 1995.

ARENDT, Hannah. La tradición oculta. Barcelona: Paidós, 2004.

ARENDT, Hannah. La condición humana. Barcelona: Paidós, 2005.

BENJAMIN, Walter. Sobre el concepto de historia. En: BENJAMIN, Walter. Obras: I, 2. Madrid: Aldaba, 2009.

BILLIG, Michael. Banal Nationalism. London: Sage, 1995.

BOURDIEU, Pierre. Les Règles de l'art. Gènese et structure du cham littéraire. Paris: Seuil, 1998.

BOURDIEU, Pierre; WACQUANT, Loïc. Las argucias de la razón imperialista. Barceona: Paidós, 1998.

BUXTON, Ellen. Historical Causation: Counter-factual Reasoning and Comparison. En: CUNSELL, C.; BURN, K.; CHAPMAN, A. (Eds.). MasterClass in History Education. Teaching and Learning. London: Bloomsbury, 2016, p. $23-42$.

CARRETERO, Mario, ASENSIO, Mikel; RODRÍGUEZ-MONEO, María. History Education and the Construction of National Identity. Charlotte: Information Age Publishing, 2013.

CASPISTEGUI, Francisco Javier. Sobre el papel social del historiador o ¿para qué servimos? Memoria y Civilización, Pamplona, n. 6, 2003, p. 191-207.

CATROGA, Fernando. Os passos do homem como restolho do tempo: memória e fim do fim da história. Coimbra: Almedina, 2009.

CERTEAU, Michel de. La escritura de la historia. México: Universidad Iberoamericana, 1993.

CHAPMAN, Arthur. Historical interpretations. En: DAVIS., I. (Ed.). Debates in History Teaching. London: Routledge, 2011, p. 96-108.

CHAPMAN, Arthur. Developing Historical and Metahistorical Thinking in History Classrooms: Reflections on Research and Practice in England. En: PRATS, J.; BARCA, I.; LÓPEZ., R. (Eds.). Historia e identidades culturales. CEID-Universidade do Minho, 2013, p. 15-36. 
CRUZ, Manuel. Adiós, historia, adiós. El abandono del pasado en el mundo actual. Oviedo: Nobel, 2012.

DE LA MONTAÑA, Juan Luis. Didáctica de la Historia y profesorado en formación: análisis de concepciones y nuevos paradigmas para una enseñanza-aprendizaje de la Historia. En: PAGÉS, J.; SANTIESTEBAN, A. (Coords.). Una mirada al pasado y un proyecto de futuro: investigación e innovación en didáctica de las ciencias sociales, vol. 2. Barcelona: Universidad Autónoma de Barcelona, 2014, p. 551-558.

DE LA MONTAÑA, Juan Luis. Didáctica de la Historia, historiografía y la visibilización de temas, grupos sociales y personas en la enseñanza-aprendizaje de la Historia. En: HERNÁNDEZ CARRETERO, A. M.; GARCÍA RUIZ, C. R.; DE LA MONTAÑA, J. L. (Coords.). Una enseñanza de las ciencias sociales para el futuro: recursos para trabajar la invisibilidad de personas, lugares y temáticas. Cáceres: UEX, 2015, p. 907-915.

DE LA MONTAÑA, Juan Luis. Didáctica de la historia y epistemología disciplinar. Notas sobre el debate y propuestas de intervención en la formación inicial del profesorado. Clio: History and History Teaching, Madrid, n. 42, 2016.

DE LA MONTAÑA, Juan Luis; RINA, César. Los modelos temáticos-diacrónicos en la enseñanza de la historia y la adquisición de competencias. En: D. Verdú, C., Guerrero y J. L. Villa. (Eds.). Pensamiento histórico y competencias sociales y cívicas en Ciencias Sociales. Murcia: Editum, 2018, p. 67-80.

DOSSE, François. L'Histoire. Paris: Armand Collin, 2000.

DUMOULIN, Olivier. Le rôle social de l'historien: de la chaire au prétoire. Paris: Albin Michel, 2003.

ELSTER, Jon. La explicación del comportamiento social. Barcelona: Gedisa, 2010.

EVANS, Richard J. Contrafactuales: ¿y si todo hubiera sido diferente? Madrid: Noema, 2017.

FEDICARIA. Contra las virtudes del progreso, las razones de la crítica. Revista Con-Ciencia Social, Sevilla, n. 18, 2014, p. 7-10.

FERGUSON, Niall. (Dir.). Historia virtual: ¿qué hubiera pasado si...? Madrid: Taurus, 1998. FONTANA, Josep. Historia: análisis del pasado y proyecto social. Barcelona: Crítica, 2000. 
FOUCAULT, Michel. Truth, Power, Self. En: FOUCAULT, Michel. Dits et écrits II. Paris: Gallimard, 2001.

GIL DE ZÁRATE, Antonio. De la instrucción pública en España. Madrid: Colegio de SordoMudos, 1855.

GIMENO LORENTE, Paz. Didáctica crítica y comunicación. Un diálogo con Habermas y la escuela de Frankfurt. Madrid: Ministerio de Educación-Octaedro, 2009.

GODINHO, Paula. O futuro é para sempre: experiência, expectativa e práticas possíveis. Através, 2017.

GÓMEZ, Cosme J.; MIRALLES, Pedro. ¿Pensar históricamente o memorizar el pasado? La evaluación de los contenidos históricos en la educación obligatoria en España. Revista de Estudios Sociales, Bogotá, n. 52, 2015, p. 42-48.

GÓMEZ, Cosme J., ORTUÑO, Jorge; MOLINA, Sebastián. Aprender a pensar históricamente: retos para la historia en el siglo XXI. Tempo e Argumento, Florianópolis, n. 6, v.11, 2014, p. 1-25.

GÓMEZ, Cosme. J.; RODRíGUEZ, Raimundo; MIRALLES, Pedro. La enseñanza de la Historia en Educación Primaria y la construcción de una narrativa nacional. Un estudio sobre exámenes y libros de texto en España. Perfiles educativos, Ciudad de México, n. 37. v.150, 2015, p. 20-38.

HABERMAS, Jürgen. Ciencia y técnica como ideología. Madrid: Tecnos, 2007.

HARTOG, François. Régimes d'historicité: présentisme et expériences du temps. Paris: Seuil, 2003.

HEGEL, Georg Wilhelm Friedrich. Lecciones sobre filosofía de la historia universal. Valencia: UVA, 1992.

HÖLSCHER, Lucian. El descubrimiento del futuro. Madrid: Siglo XXI, 2014.

KLEIN, Olivier. The lay historian: how ordinary people think about history. En:

CABECINHAS, R.; ABADIA, L. (Eds.). Narrative and social memory: theoretical and methodological approaches. Braga: University of Minho, 2013, p. 23-45.

KOSELLECK, Reinhart. Der zufall als motivationsrest in der geschichtsschreibung. En: Vergangene zukunft, zur semantik geschichtlicher zeiten. Fráncfort: Suhrkamp, 1979.

KOSELLECK, Reinhart. Historia magister vitae. En: Futuro pasado: para una semántica de los tiempos históricos. Barcelona: Paidós, 1993, p. 21-66. 
KOSELLECK, Reinhart. Historia/historia. Madrid, Trotta, 2010.

KOSELLECK, Reinhart. Historias de conceptos: estudios sobre semántica y pragmática del lenguaje político y social. Madrid: Trotta, 2012.

LÉVESQUE, Stéphane. Thinking historically: educating students for the twenty-first century. Toronto: Toronto University Press, 2008.

LÓPEZ, César. Conceptos y narrativas sobre la nación en estudiantes y en historiadores. Tesis Doctoral en Historia - Universidad Complutense de Madrid, Madrid, 2012.

LÓPEZ FACAL, Ramón; SÁIZ, Jorge. Spain: history education and nationalism conflicts. En: GUYVER, R. (Ed.). Teaching history and the changing nation state: transnational and international perspectives. London: Bloomsbury, 2016, p. 201-215.

LOWENTHAL, David. El pasado es un país extraño. Madrid, Akal, 1998.

MACINTYRE, Alasdair. Causalidad e historia. En: MANNINEN J.; TOUMELA, R. (Eds.). Ensayos sobre explicación y comprensión. Madrid: Alianza, 1980.

MARQUARD, Odo. Apología de lo contingente. Valencia: Institución Alfonso el Magnánimo, 2000.

MIRALLES, Pedro; GÓMEZ, Cosme J. Enseñanza de la historia, análisis de libros de texto y construcción de identidades colectivas. Historia y Memoria de la Educación, Madrid, n. 6, 2017, p. 9-28.

MOLINA, Sebastián; MIRALLES, Pedro, DEUSDAD, Blanca; ALFAGEME, María Begoña. Enseñanza de la historia, creación de identidades y prácticas docentes. Profesorado:

Revista de Currículum y Formación del profesorado, Granada, v. 21, n. 2, 2017, p. 331-354.

MORO, Oscar. La recuperación de lo contingente: Foucault y Bourdieu. En: ROLDAN, C.; MORO, O. (Eds.). Aproximaciones a la contingencia histórica y actualidad de una idea. Madrid: Catarata, 2009, p. 131-149.

NISBERT, Robert. Historia de la idea de progreso. Barcelona: Gedisa, 1996.

NORA, Pierre. Présent, nation, mémoire. Paris: Gallimard, 2011.

ORTUÑO, Jorge, PONCE, Ana Isabel; SERRANO, Francisco José. La idea de causalidad en las explicaciones históricas del alumnado de educación primaria. Revista de Educación, Madrid, n. 371, 2016, p. 9-34. 
PARKES, Roberts. Teaching history as historiography: engaging narrative diversity in the curriculum. International Journal of Historical Learning, Teaching and Research, Carlisle, v. 8, n. 2, 2009, p. 118-132.

PARRA, David; SEGARRA, Josep Ramón. El aprendizaje de la nación en los regímenes pedagógicos de la España contemporánea. En: QUIROGA, A.; ARCHILÉS, F. (Eds.). Ondear la nación: nacionalismo banal en España. Granada: Comares, 2018, p. 181-204.

PÉREZ GARZÓN, Juan Sisinio. ¿Por qué enseñamos Geografía e Historia? ¿Es tarea educativa la construcción de identidades? Historia de la Educación, n. 27, 2008, p. 37-55.

PÉREZ GARZÓN, Juan Sisinio. Entre la historia y las memorias: poderes y usos sociales en juego. En: PÉREZ GARZÓN, Juan Sisinio; Manzano, E. (Eds.). Memoria Histórica. Madrid: CSIC-Catarata, 2010, p. 23-70.

POPPER, Karl R. La miseria del historicismo. Madrid: Taurus, 1961.

POPPER, Karl R. El desarrollo del conocimiento científico: conjeturas y refutaciones. Buenos Aires: Paidós, 1967.

RICOUER, Paul. La Lectura del tiempo pasado: historia y olvido. Madrid: Arrecife, 1999.

RICOEUR, Paul. Tiempo y narración: configuración del tiempo en el relato histórico. Tomo I. México: Siglo XXI, 2013.

RINA, César. De la Historia sólida a las historias líquidas. Los condicionantes tecnológicos y neoliberales del oficio. Diacronie - Studi di Storia Contemporanea, Bologna, v.4, n. 12, 2012, p. 1-15.

RINA, César. La didáctica de la historia en los procesos de nacionalización. trayectoria, constantes y propuestas. CLIO. History and History teaching, Madrid, n. 43, 2017, p. 225242.

RINA, César; CLEMENTE, Luis Vicente. Usos públicos de la historia en la España contemporánea: nacionalismos, regionalismos y modelos de estado. En: COLOMER, J. C.; ESTEVE, J.; IBÁÑEZ, M. (Coords.). Ayer y hoy: debates, historiografía y didáctica de la historia. Valencia: AHC-UVA, 2015, p. 103-107.

ROBIN, Régine. La mémoire saturée. Paris: Stock, 2003.

ROHBECK, Johannes. Sobre el trato razonable con la contingencia histórica. En: ROLDÁN, C.; MORO O. (Eds.). Aproximaciones a la contingencia: historia y actualidad de una idea. Madrid: Catarata, 2009, p. 205-220. 
ROLDÁN, Concha; MORO, Oscar. (Eds.). Aproximaciones a la contingencia: historia y actualidad de una idea. Madrid: Catarata, 2009

RORTY, Richard. Contingencia, ironía y solidaridad. Barcelona: Paidós, 1991.

RÜSSEN, Jörn. Didáctica da história: passado, presente e perspectivas a partir do caso alemão. Praxis Educativa, Santa Rosa, v. 1, n. 2, 2006, p. 7-16.

RÜSSEN, Jörn. Historiología: esquema de una teoría de la historiología. Memoria y Civilización, Pamplona, n. 15, 2012, p. 415-447.

SAFRANSKI, Rüdiger. Tiempo: la dimensión temporal y el arte de vivir. Barcelona: Tusquets, 2017.

SAIZ, Jorge y LÓPEZ FACAL, Ramón. Aprender y argumentar España. La visión de la identidad española entre el alumnado al finalizar bachillerato. Didáctica de las Ciencias Experimentales y Sociales, Valencia, n. 26, 2012, p. 95-120.

SAIZ, Jorge y LÓPEZ FACAL, Ramón. Narrativas nacionales históricas de estudiantes y profesorado en formación. Revista de Educación, Madrid, n. 374, 2016, p. 118-141.

SAIZ, Jorge; GÓMEZ, Celso J. ; LÓPEZ FACAL, Ramón. Historical thinking, causal explanation and narrative discourse in trainee teachers in Spain. Historical Encounters, Callaghan, n. 5, 2018, p. 16-30.

SANTOS UNAMUNO, Enrique. Ficción televisiva y estereotipos (inter)nacionales: un acercamiento imagológico a El Ministerio del Tiempo (2015-2016). Amnis - Revue civilisation contemporaine Europes/Amériques, Aix en Provence, n. 17, 2018.

SEIXAS, Peter; MORTON, Tom. The big six historical thinking concepts. Toronto: Nelson, 2013.

TODOROV, Tzvetan. Los abusos de la memoria. Madrid: Paidós, 2008.

TRAVERSO, Enzo. El pasado, instrucciones de uso: historia, memoria, política. Madrid: Marcial Pons, 2007.

VANSLEDRIGHT, Bruce A. The challenge of rethinking history education: on practice, theories and policy. New York: Routledge, 2011.

WINEBURG, Sam. Historical thinking and other unnatural acts: charting the future of teaching the past. Philadelphia: Temple University Press, 2001.

ZERMEÑO, Guillermo. La cultura moderna de la historia: una aproximación teórica e historiográfica. México: Colegio de México, 2010. 
ZERMEÑO, Guillermo. (Ed.). Historia fin de siglo. México: Colegio de México, 2016. 